\title{
Energetics of large carbon clusters: Crossover from fullerenes to nanotubes
}

\author{
Noejung Park, ${ }^{1}$ Kyuho Lee, ${ }^{1,2}$ Seungwu Han, ${ }^{2,3}$ Jaejun Yu, ${ }^{1,2,}{ }^{*}$ and Jisoon Ihm ${ }^{1}$ \\ ${ }^{1}$ School of Physics, Seoul National University, Seoul 151-742, Korea \\ ${ }^{2}$ Center for Strongly Correlated Materials Research, Seoul National University, Seoul 151-742, Korea \\ ${ }^{3}$ Princeton Materials Institute, Princeton University, Princeton, New Jersey 08544
}

(Received 12 December 2001; published 12 March 2002)

\begin{abstract}
The energetics of large-sized fullerenes and carbon nanotubes is investigated through first-principles pseudopotential calculations for the carbon cluster of $\mathrm{C}_{N}(60 \leqslant N \leqslant 540)$. The strain energy due to the presence of pentagons, in addition to the curvature effect, makes an important contribution to the energetics of the fullerenes and nanotubes and accurately describes the $N$ dependence of the energy of the spherical fullerenes. Our model predicts that a nanotube of $\sim 13 \AA$ in diameter [for example, a $(9,9)$ or $(10,10)$ tube] is energetically most stable among various single-walled nanotubes and fullerenes, consistent with many experimental observations.
\end{abstract}

DOI: 10.1103/PhysRevB.65.121405

PACS number(s): 73.22.-f, 61.48.+c, 71.15.Nc

Since the discovery of carbon nanotubes, ${ }^{1}$ a great deal of progress has been made in research of carbon nanotubes for their technological applications, such as nanoelectronic devices, probing tips, electron emitters, etc. Among various forms of carbon nanotubes or clusters in nanoscales, the single-walled nanotube has attracted much attention because of its unique structural and electrical properties. However, the study of single-walled nanotubes has often been hindered by the lack of knowledge in the basic growth mechanism of carbon nanotubes and related clusters. Recent experiments ${ }^{2-7}$ have reported that the diameters of the single-walled carbon nanotubes have a narrow distribution range around that of the $(10,10)$ tube. Several models of the nanotube growth ${ }^{8-10}$ have suggested that the formations of the single-walled nanotubes and fullerenes are initiated by graphitic bowls, and the energetics of large carbon clusters should play a crucial role. While a single-walled nanotube is frequently described as a rolled-up graphitic sheet, it may as well be considered as an elongated fullerene when it is capped. Since the fullerene itself is another important form of carbon cluster, the energetics of large fullerenes in general, including the capped nanotubes, should be useful in understanding the initial growth process of nanotubes and this diameter distributions.

In an earlier work, Adams and co-workers ${ }^{11}$ showed that a key factor in determining the energetics of large fullerenes and carbon nanotubes was the nonplanarity of the graphitic sheet incurring incompleteness of the $\pi$ bonding. Based on the empirical formula derived from the computational results, they concluded that the spherical fullerenes were energetically favored over the tubelike elongated fullerenes, i.e., capped nanotubes. Since, however, theoretical studies of large carbon clusters demand enormous electronic structure calculations, the fully self-consistent first-principles calculations have been restricted to relatively small system size. So far many calculations on the large carbon clusters were done by classical continuum theory, ${ }^{12}$ molecular dynamics with the three-body potential, ${ }^{13}$ or so-called $O(N)$ methods with non-self-consistent Harris functional formalism. ${ }^{11,14,15}$

In this work, we have performed self-consistent firstprinciples calculations to obtain fully relaxed equilibrium geometries and corresponding total energies of the spherical fullerenes and the capped nanotubes of $\mathrm{C}_{N}(60 \leqslant N \leqslant 540)$. Through the analysis of the total-energy results on the spherical fullerenes as well as capped nanotubes, we conclude that the strain energy due to the presence of pentagons, in addition to the curvature energy of the graphitic sheet, makes an important contribution to the energetics of largesized $s p^{2}$ carbon clusters. We construct a model formula for the energy of capped nanotubes and fullerenes, which takes account of both the curvature and pentagon strain contributions.

Aiming at the electronic structure calculations of largesized systems, we have developed a self-consistent firstprinciples calculational method based on density-functional formalism ${ }^{16}$ within a local-density approximation. We adopt the numerical basis set for the localized pseudoatomic orbitals (one $s$ and three $p$ orbitals per atom), which is generated through the method of Sankey and Niklewski. ${ }^{17}$ The pseudopotential is generated through the Troullier-Martins scheme ${ }^{18}$ and its nonlocal part is converted into the Kleinman-Bylander ${ }^{19}$ type of the fully separable form. In this work, we used the Ceperley-Alder form of the exchangecorrelation potential. ${ }^{20}$ The minimum-energy configuration is obtained through the relaxation of the atomic force which includes the Pulay-like correction ${ }^{21}$ to the Hellman-Feynman force. The detailed description of our method will be published elsewhere. ${ }^{22}$ For the comparison of our localized-basis method with the standard plane-wave method, we calculate cohesive energies for small-sized fullerenes and compare the results as shown in Fig. 1. We use the same geometric structures of fullerenes given in the literature, ${ }^{23,24}$ and set the energies of $\mathrm{C}_{32}$ as a reference for comparison. The size dependence of the total energies of the fullerene is quite accurately reproduced with this minimal basis set, and further the relative values of the cohesive energy are in good agreement with the results reported by other groups. ${ }^{23,24}$

To investigate the energetics of large fullerenes $\mathrm{C}_{N}$, we carried out total-energy calculations with a full relaxation of atomic positions within a given geometry for $N$ $=60,80, \ldots, 540$. Among many different faceted or irregular shapes of fullerenes, we selected spherical ones (or "icosahedral" fullerenes) which were found to be stable. ${ }^{14,15}$ The 


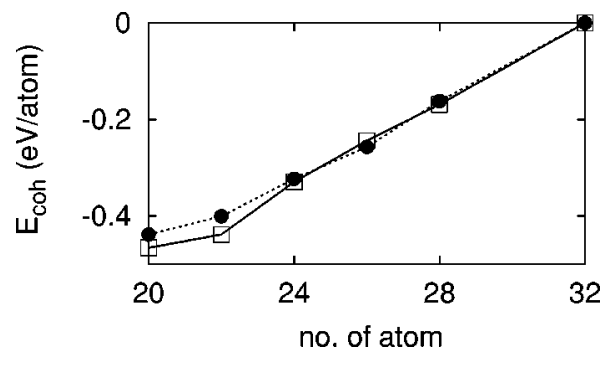

FIG. 1. Comparison of the calculated cohesive energies of small fullerenes. Filled circles are the results of the standard plane-wave method and squares are the results of the localized-basis method.

calculated total energies with respect to that of the infinite graphene sheet are presented in Fig. 2(b). The energetics of the spherical fullerene here shows somewhat different results from the prediction of the previous work, ${ }^{11}$ which is denoted as a dotted line in Fig. 2(b), and stays flat in the range of $N>240$. As discussed later in this paper, we describe the energetics of the spherical fullerene by noting two different strain sources: one from the curvature (nonplanarity) and the other from the bond-length change of the pentagons.

In order to understand the pure curvature effect without pentagons, we first investigate the energetics of the carbon nanotubes. Figure 2(a) shows the total energy of the $(n, n)$ carbon nanotubes without edges, i.e., infinitely long tubes. As pointed out in Ref. 11, the curvature energy in the hexagonal network of carbons can be modeled by small hybridization between $s p^{2}$ orbitals and $p_{z}$ orbitals. Since the interaction between two nearly parallel neighboring $\pi$ orbitals is proportional to $\cos \phi_{\pi}$, where $\phi_{\pi}$ is the average angle between two neighboring $\pi$ orbitals, the curvature energy per atom can be described by ${ }^{11}$

$$
\epsilon_{\text {tube }}=\epsilon\left[1-\cos \phi_{\pi}\right] \text {. }
$$

Here the magnitude of $\epsilon$ may depend (very slightly) on the chirality of tubes, while the size dependence of the energy is described by the parameter $\phi_{\pi} .{ }^{11}$ For the $(n, n)$ nanotubes with large $n$, the angle $\phi_{\pi}$ is proportional to the ratio of the

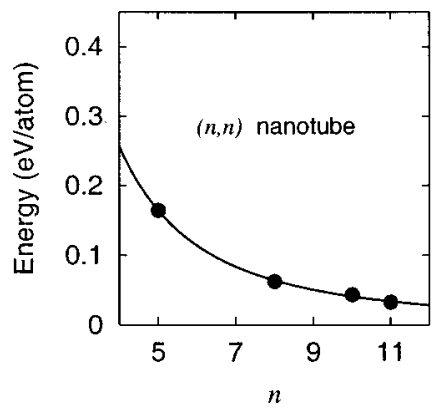

(a)

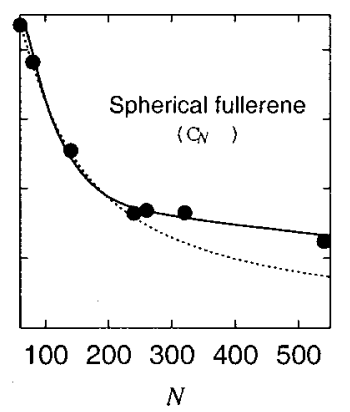

(b)
FIG. 2. Total energy of (a) the carbon nanotubes and (b) the spherical fullerenes with respect to the infinite graphene sheet. Filled circles are the total energies from the self-consistent calculations, and the solid lines are the fitted curves of our strain energy model described in the text. The dotted line in (b) is the result of a previous work (Ref. 11).

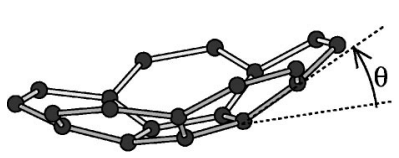

(a)

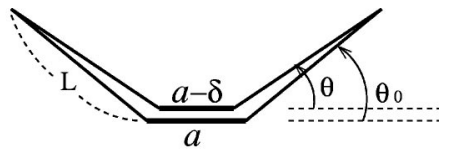

(b)
FIG. 3. (a) A portion of the graphitic cone structure. The cone angle $\theta$ is defined to be the angle between the pentagonal plane and the $\mathrm{C}-\mathrm{C}$ bond which stems from the vertex of the pentagon. (b) A schematic illustration of the bond-length change of the pentagon (thick horizontal bars) accompanied by the cone angle change.

C-C bond length $d$ over the tube radius $r$, i.e., $d / r$. Since the tube index $n$ is proportional to $r$, we have the relation $\phi_{\pi}$ $\propto(1 / n)$. Therefore, the curvature energy of the nanotube (per atom) can be written in the form

$$
\epsilon_{\mathrm{tube}} \approx \epsilon^{\prime}\left(\frac{d}{r}\right)^{2}=\frac{\epsilon_{1}}{n^{2}}
$$

Indeed the results of our first-principles calculations on the $(n, n)$ tubes are extremely well fitted to this simple formula with a parameter $\epsilon_{1}=4.12 \mathrm{eV}$, as indicated by the solid line in Fig. 2(a). The value of $\epsilon_{1}=4.12 \mathrm{eV}$ is very close to the result of the previous work. ${ }^{11}$

Similarly, the curvature energy $\left(\epsilon_{\mathrm{cf}}\right)$ of the spherical fullerene (per atom) can be modeled as

$$
\epsilon_{\mathrm{cf}}=\epsilon\left(1-\cos \phi_{\pi}\right) \approx \epsilon^{\prime \prime}\left(\frac{d}{r}\right)^{2}=\frac{\epsilon_{2}}{N},
$$

where $r$ is the radius of the fullerene. Note that the surface area of the fullerene $\left(4 \pi r^{2}\right)$ is proportional to the total number of atoms $N$. In Fig. 2(b), it is obvious that the calculated $N$ dependence of the strain energy deviates significantly from the simple formula of Eq. (3), implying that the curvature effect alone is not sufficient for the description of the energetics of fullerenes.

Another important contribution to the total energy of the fullerenes distinct from the curvature effect has been known to come from the strain in the pentagon region. The origin of such strain can be sought in the geometry of an isolated graphitic cone. A cone here is defined to be a structure where a pentagon is surrounded by a hexagonal network. The cone angle $\theta$ of the isolated graphitic cone in Fig. 3(a) is found to be about $26^{\circ}$ in its equilibrium configuration, and the corresponding nonplanarity angle $\psi$ of the pentagon ${ }^{25}$ is $8.2^{\circ}{ }^{26}$ If the cone angle $\theta$ is forced to deviate from its equilibrium value $\theta_{o}$, an extra energy which we call "pentagonal strain energy" occurs. While the curvature energy arises from the orbital hybridization, the pentagonal strain energy comes from the change of the C-C bond lengths from the values of the equilibrium configuration. Through extensive $a b$ initio calculations, we find that the pentagonal strain energy can be modeled by assuming that the bond-length change is effectively confined to the five bonds forming the pentagon. As shown in the schematic illustration of Fig. 3(b), the bondlength change of the pentagon $(\delta)$ is related to the length of 
the cone edge $(L)$ and the cone angle change $\left(\theta-\theta_{o}\right)$, which is assumed to be small, as follows:

$$
\delta=2 L\left(\cos \theta-\cos \theta_{o}\right) \propto \sqrt{N_{\text {cone }}}\left(\theta-\theta_{o}\right) .
$$

The length $L$ of the cone edge is approximately proportional to the square root of the number of atoms belonging to the cone $\left(N_{\text {cone }}\right)$. Note that $L$ is assumed to remain the same and only the pentagon bond length ( $a$ in the figure) changes.

Now we describe the spherical fullerene as 12 interconnected cones through the intermediate graphitic (i.e., hexagonal) region. The total strain energy of 12 cones can be expressed as

$$
E_{\text {cone }}=12 \times \frac{\kappa^{\prime}}{2} N_{\text {cone }}\left(\theta-\theta_{o}\right)^{2} \approx 12 \times \frac{\kappa}{2} N_{\text {cone }}\left(\frac{1}{\sqrt{N}}-\frac{1}{\sqrt{N_{0}}}\right)^{2},
$$

where we assume that the fullerenes are nearly spherical and, hence, $\theta \propto 1 / r$ and $r \propto \sqrt{N}$. Our calculations indicate that the best choice of $N_{\text {cone }}$ is 20 as shown in Fig. 3(a). This is valid whenever $N \geqslant 240$, i.e., when $N$ exceeds the total number of atoms participating in the 12 cones. However, if $N<240$, the size of each cone should shrink because the total number of atoms participating in the 12 cones cannot exceed $N$. Thus we set $N_{\text {cone }}$ as follows,

$$
12 \times N_{\text {cone }}= \begin{cases}N & \text { if } N<240 \\ 240 & \text { otherwise }\end{cases}
$$

Now we construct an energy model for the spherical fullerene as follows:

$$
\epsilon_{\mathrm{fl}}=\frac{1}{N}\left[(N-60) \epsilon_{\mathrm{cf}}+E_{\text {cone }}\right]
$$

Here we assume that, based on the first-principles calculational results with relaxations, the pentagon itself remains as a regular polygon independently of the size of the fullerene. Hence we ignore the angle change between the $\pi$ orbital within the pentagon and the corresponding curvature energy. We only consider the curvature energy in the $(N-60)$ atoms not participating in the pentagons and $E_{\text {cone }}$ which reflects the energy associated with the bond-length change of the pentagon as well as the angle change between the $\pi$ orbitals of the pentagon and that of the neighbors. The parameters in Eq. (7) are obtained through the least-squares fit to the calculated total energies for $N=60,80,140,240,260$, and 320, and the results are $\kappa=21.9 \mathrm{eV}, N_{0}=200$, and $\epsilon_{2}$ $=55.1 \mathrm{eV}$. To check the validity of our fit, we have calculated an extra point at $N=540$ not included in the fitting procedure. The result for $N=540$ shown in Fig. 2(b) proves the quality of our energy model. From the model, it is recognized that the curvature effect and the pentagonal strain effect operate in the same direction for small-sized fullerenes so that the spherical fullerene becomes more stable. In a larger fullerene, on the other hand, the two strain sources compete and the relatively flat region develops beyond $N$ $\approx 200$ in the energy curve.

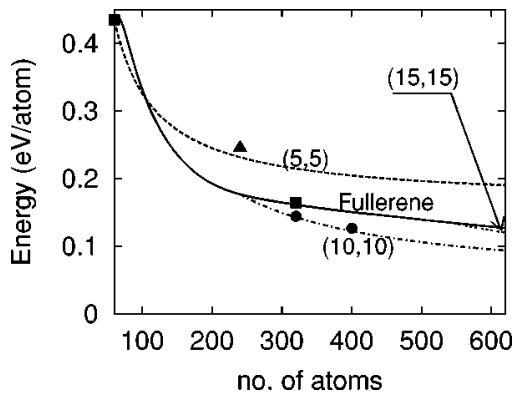

FIG. 4. The $N$ dependence of the energy for the capped $(n, n)$ nanotubes [dashed line for $(5,5)$, dash-dotted line for $(10,10)$, and dotted line for $(15,15)$ tubes] in comparison with spherical fullerenes(solid line). The triangle, squares, and circles correspond to the total energies from our self-consistent calculations for the $(5,5)$ tube, fullerenes $(N=60$ and $N=320)$, and the $(10,10)$ tubes, respectively.

The equilibrium geometries obtained from our ab initio calculations confirm the assumptions employed in the energy model. As the size of the fullerene increases, the bond-length change is the most significant in the pentagon: The pentagon bond length monotonically decreases in the range $60 \leqslant N$ $\leqslant 320$ (from $1.486 \AA$ of $\mathrm{C}_{60}$ to $1.426 \AA$ of $\mathrm{C}_{320}$ ). Among them, $\mathrm{C}_{140}$ and $\mathrm{C}_{240}$ have the value of the bond length close to the unstrained graphite $(1.452 \AA)$. The cone angle $\theta$ of $\mathrm{C}_{240}$ is about $26^{\circ}$, corresponding to the angle of the unstrained isolated cone geometry, whereas that of the $\mathrm{C}_{60}$ is about $32^{\circ}\left(\psi=11.9^{\circ}\right)$. These results are in good agreement with the results of Ref. 25.

Now we are going to construct the energy model for the single-walled $(n, n)$ nanotubes with caps. Since most singlewalled nanotubes are closed with a fullerenelike cap, ${ }^{4,3}$ the energy of the capped tubes is more relevant to experiments than open tubes. Here, we assume the cap of nanotubes is a hemisphere of spherical fullerenes; for example, the cap of the $(5,5)$ tube is the hemisphere of $\mathrm{C}_{60}$, and the cap of the $(10,10)$ tube is that of $C_{240}$. By summing up the energy contributions from the pure tube region of $\left(N-N_{\text {cap }}\right)$ atoms and the fullerenelike cap regions of $N_{\text {cap }}$ atoms, the total energy per atom is

$$
\epsilon_{\text {cap-tube }}=\left(\frac{N-N_{\text {cap }}}{N}\right) \epsilon_{\text {tube }}+\left(\frac{N_{\text {cap }}}{N}\right) \epsilon_{\text {fl }} .
$$

This expression agrees with independent total-energy calculations for three capped tube geometries [one $(5,5)$ tube and two $(10,10)$ tubes], as shown in Fig. 4. Contrary to the prediction of the previous work, ${ }^{11}$ the energy curve of the $(10,10)$ nanotube stays below that of the fullerene. This fact indicates that the $(10,10)$ nanotube is not only more stable than the fullerenes, but it is more stable than smaller or even larger nanotubes as well. ${ }^{27}$ Since the total energy of the same-diameter tube is insensitive to the chirality in known cases, ${ }^{11}$ the capped nanotubes with the same diameter but different chirality should be as stable as the $(10,10)$ nanotube when they are well matched with the cap without significant bond distortions. Thus, for example, the $(18,0)$ nanotube capped with a hemisphere of $\mathrm{C}_{240}$, which keeps the sixfold 
rotational symmetry, can be as stable as the $(10,10)$ nanotube. Our energy model suggests that the decisive factor of the diameter distribution of the single-walled nanotube is the energetics of the cap (or graphitic bowl) at the initial stage of the formation. For instance, if we consider a graphitic bowl of a few hundred of carbon atoms $(N \sim 320)$, the total energy of the $(10,10)$ tubelike elongated fullerene is lower than its isomeric spherical fullerene by about $1 \mathrm{Ry}$.

In summary, we have presented the energetics of the spherical fullerenes and nanotubes as obtained from fully self-consistent first-principles calculations on large carbon clusters $\mathrm{C}_{N}(60 \leqslant N \leqslant 540)$, and constructed the fitting model for them. While the energetics of the long nanotubes can be well described in terms of the curvature effect, understanding of the spherical fullerenes or the cap of the nanotubes requires an additional term, namely, the pentagonal strain attributed to the change of the $\mathrm{C}-\mathrm{C}$ bond lengths in the penta- gon. The $N$ dependence of energy in our model explains the retardation of the rate of the energy decrease in the range of $N \geqslant 240$ [Fig. 2(b)] for the case of spherical fullerenes, which is the consequence of the competition between the curvature effect and the pentagonal strain effect. Our prediction that the capped $(10,10)$ carbon nanotube is more stable than fullerenes or other (smaller- or larger-diameter) nanotubes is consistent with experimental observations. ${ }^{2-7}$

This work was supported by Samsung Electronics, the Samsung Alpha-Cluster Project, and the Supercomputing Application Support Program of KISTI. J.Y. acknowledges the support of the KOSEF through CSCMR, and N.P. and J.I. acknowledge the support of the KOSEF through CNNC. Helpful discussions with Professor R. Car are greatly appreciated.
*Email: jyu@snu.ac.kr

${ }^{1}$ S. Iijima, Nature (London) 354, 56 (1991).

${ }^{2}$ S. Iijima and T. Ichihashi, Nature (London) 363, 603 (1993).

${ }^{3}$ C.-H. Kiang and W.A. Goddard III, Phys. Rev. Lett. 76, 2515 (1996).

${ }^{4}$ A. Thess, R. Lee, P. Nikolaev, H. Dai, P. Pett, J. Robert, C. Xu, Y.H. Lee, S.G. Kim, A.G. Rinzler, D.T. Colbert, G.E. Scuseria, D. Tomónek, J.E. Fischer, and R.E. Smalley, Science 273, 483 (1996).

${ }^{5}$ J.M. Cowley, P. Nikolaev, A. Thess, and R.E. Smalley, Chem. Phys. Lett. 265, 379 (1997).

${ }^{6}$ S. Bandow, S. Asaka, Y. Saito, A.M. Rao, L. Grigorian, E. Richter, and P.C. Eklund, Phys. Rev. Lett. 80, 3779 (1998).

${ }^{7}$ Y. Zhang, H. Gu, and S. Iijima, Appl. Phys. Lett. 73, 3827 (1998).

${ }^{8}$ H. Kanzow, C. Lenski, and A. Ding, Phys. Rev. B 63, 125402 (2001).

${ }^{9}$ S.V. Rotkin and R.A. Suris, Phys. Lett. A 261, 98 (1999).

${ }^{10}$ A. Krishnan, E. Dujardin, M.M.J. Treacy, J. Hugdahl, S. Lynum, and T.W. Ebbesen, Nature (London) 388, 451 (1997).

${ }^{11}$ G.B. Adams, O.F. Sankey, J.B. Page, M. O'Keeffe, and D.A. Drabold, Science 256, 1792 (1992).

${ }^{12}$ D. Tománek, W. Zhong, and E. Krastev, Phys. Rev. B 48, 15461 (1993).

${ }^{13}$ A. Maiti, C.J. Brabec, and J. Bernholc, Phys. Rev. Lett. 70, 3023 (1993)

${ }^{14}$ D. York, J.P. Lu, and W. Yang, Phys. Rev. B 49, 8526 (1994).
${ }^{15}$ J.P. Lu and W. Yang, Phys. Rev. B 49, 11421 (1994).

${ }^{16}$ P. Hohenberg and W. Kohn, Phys. Rev. 136, B864 (1964); W. Kohn and L.J. Sham, Phys. Rev. 140, A1133 (1965).

${ }^{17}$ O.F. Sankey and D.J. Niklewski, Phys. Rev. B 40, 3979 (1989).

${ }^{18}$ N. Troullier and J.L. Martins, Phys. Rev. B 43, 1993 (1991).

${ }^{19}$ L. Kleinman and D.M. Bylander, Phys. Rev. Lett. 48, 1425 (1982).

${ }^{20}$ D.M. Ceperley and B.J. Alder, Phys. Rev. Lett. 45, 566 (1980).

${ }^{21}$ M. Scheffler, J.P. Vigneron, and G.B. Bachelet, Phys. Rev. B 31, 6541 (1985).

${ }^{22}$ N. Park, K. Lee, S. Han, J. Yu, and J. Ihm (unpublished).

${ }^{23}$ R.O. Jones and G. Seifert, Phys. Rev. Lett. 79, 443 (1997).

${ }^{24}$ P.R.C. Kent, M.D. Towler, R.J. Needs, and G. Rajagopal, Phys. Rev. B 62, 15394 (2000).

${ }^{25}$ P. Ordejón, E. Artacho, and J.M. Soler, Phys. Rev. B 53, R10 441 (1996).

${ }^{26}$ The cone angle $\theta$ and the nonplanarity angle $\psi$ are related by $-\sin \pi / 5 \cos \theta=\cos [(7 \pi / 10)-(\psi / 2)]$.

${ }^{27}$ Although not shown in Fig. 4, the energy curve of the $(9,9)$ tube which starts at $N \sim 190$ nearly overlaps with that of the $(10,10)$ tube in the range $N>300$. Therefore, a more precise statement of our prediction is that the $(10,10)$ tube or a slightly smaller one, say a $(9,9)$ tube, is the most stable. The energy curve of the $(15,15)$ tube, presented as an example of a tube larger than the $(10,10)$ tube, starts at $N=540$ and stays higher than that of the $(10,10)$ tube in the range of $N$ shown in Fig. 4. 